\title{
Monitoring of Injection Moulding of Thermoplastics: Adopting Pressure Transducers to Estimate the Solidification History and the Shrinkage of Moulded Parts
}

\author{
Vito Speranza - Umberto Vietri - Roberto Pantani* \\ University of Salerno, Department of Industrial Engineering, Italy
}

\begin{abstract}
In this work, a series of injection moulding tests were conducted using a general purpose PolyStyrene, PS, and changing the holding pressure, injection temperature and cavity dimensions. The pressures at the interface with the mould at several positions along the flow-path were measured by means of pressure-temperature transducers. The samples were measured after moulding to determine dimensional accuracy, which was taken to be the target quality parameter. The pressure profiles obtained were then analysed using a recently developed procedure that is able to estimate the local solidification history from pressure measurements. The local average solidification pressure, namely the average along the thickness direction of the pressures at which each layer solidifies, could thus be estimated. This parameter is known to correlate well with shrinkage and thus a master-curve can be created that can be adopted to monitor the quality of the moulded part on line. Keywords: injection moulding, quality control, shrinkage
\end{abstract}

\section{INTRODUCTION}

The development of advanced techniques for monitoring and controlling the injection moulding process is a strategic issue for industries involved in polymer processing operations [1].

In-mould sensors can be very helpful for on line measurements and hence for monitoring and control purposes [2]. Ultrasonic [3] and capacitive [4] sensors have been applied to measure the part weight, optical fibers [5] have proven to be able to measure thickness shrinkage, and strain gages [6] and [7] have been adopted to follow the shrinkage evolution from the instant of first solidification. However, these methods are normally limited to scientific purposes: industries are traditionally disinclined to introduce moulds instrumented with a suitable number of sensors that could effectively monitor the injection moulding process. In spite of this, traditional hardware-based temperature and pressure transducers have been widely employed in industry; however, there is no clear correlation between the measured evolution of temperature and pressure and the product quality. [8].

Indeed, it can be easily demonstrated [9] that even the complete pressure curve cannot be adopted as a suitable parameter to fully describe shrinkage, and a criterion based on the reproducibility of the pressure profiles can cause the rejection of parts that are consistent with quality parameters. On the other hand, the local average solidification pressure $\overline{P_{S}}$ (the average over the thickness of the pressures at which each layer solidifies locally) was demonstrated to be a suitable parameter for quality part description in the injection moulding process [9]. Determining the local average solidification pressure $\overline{P S}$ requires the determination of both the local pressure history and the local solidification history. In spite of the recent attempts made to experimentally determine the temperature profile along the thickness direction of a moulding [10], the local solidification history is not experimentally obtainable, and thus it is necessary to perform a simulation of the whole injection moulding test in order to obtain it.

In a previous work [11], a procedure was introduced which allows the adoption of the measured pressure evolution to make an estimation of the solidification profile and thus of the average solidification pressure.

In this work the procedure was applied to injection moulding tests carried out with a general purpose PS.

\section{EXPERIMENTAL}

\subsection{Material}

The material adopted was a general purpose Polystyrene (Styron PS 678E) supplied by Dow Chemicals. A complete characterization of the resin can be found in the literature [12] to [14].

\subsection{Moulding Conditions}

Four series of experiments were carried out; each one characterized by the variation of a single parameter (namely, injection temperature and cavity thickness) with respect the reference series (series A). For each series, several holding pressures were adopted from 
80 bar up to more than 1000 bar. A summary of the moulding conditions is reported in Table 1.

For each of the moulding conditions, measurements for pressure evolution data were taken at three positions inside the cavity at 15, 60 and $105 \mathrm{~mm}$ from the gate. The cavity is $120 \mathrm{~mm}$ long and these positions (referred to as $P_{2}, P_{3}$ and $P_{4}$, respectively) are located $15 \mathrm{~mm}$ from the cavity entrance, in the center of the cavity, and at $15 \mathrm{~mm}$ from the cavity tip. The other two transducers were located inside the injection chamber (pos. $P_{0}$ ) and just upstream from the gate (pos. $P_{1}$ ). A schematic view of the cavity adopted for all the moulding tests is shown in Fig. 1.

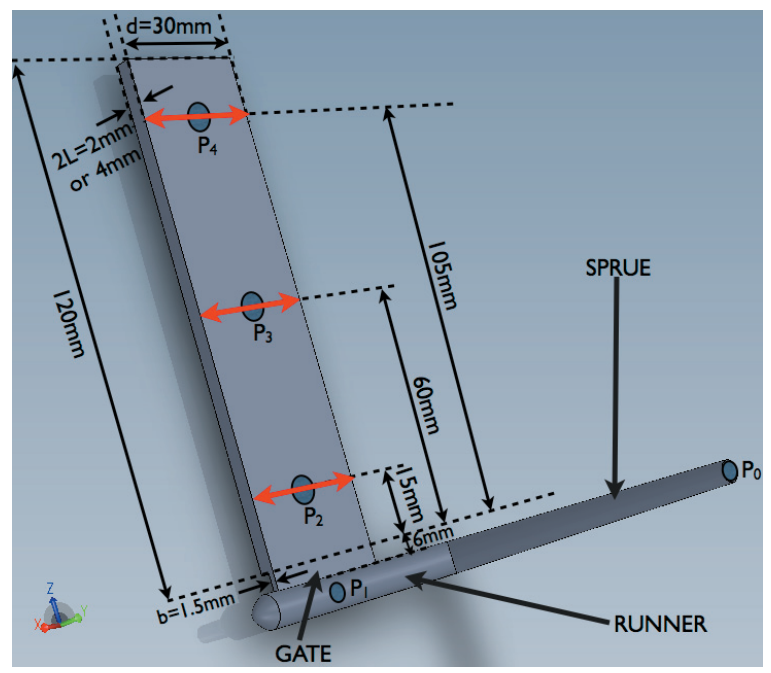

Fig. 1. Schematic view of the geometry adopted for all the moulding tests; the dimensions considered for shrinkage are indicated

Table 1. Summary of moulding conditions: for each series of experiments, the parameter characterising the series is reported in bold

\begin{tabular}{|c|c|c|c|c|c|c|}
\hline Series & $\begin{array}{c}P_{h} \\
{[\mathrm{bar}]}\end{array}$ & $\begin{array}{l}t_{h} \\
{[s]}\end{array}$ & $\begin{array}{l}t_{i n j} \\
{[\mathrm{~s}]}\end{array}$ & $\begin{array}{l}T_{i n j} \\
{\left[{ }^{\circ} \mathrm{C}\right]}\end{array}$ & $\begin{array}{c}T_{\text {mould }} \\
{\left[{ }^{\circ} \mathrm{C}\right]}\end{array}$ & $\begin{array}{l}\text { Thickness } \\
\text { [mm] }\end{array}$ \\
\hline A & 70 to 1300 & 12 & 0.45 & 200 & 25 & 2 \\
\hline$B$ & 70 to 1100 & 12 & 0.45 & 220 & 25 & 2 \\
\hline $\mathrm{C}$ & 140 to 1000 & 12 & 0.45 & 240 & 25 & 2 \\
\hline D & 90 to 1200 & 12 & 0.45 & 200 & 25 & 4 \\
\hline
\end{tabular}

$P_{h}$ stands for holding pressure, $t_{h}$ for holding time, $t_{i n j}$ for injection time, $T_{i n j}$ for injection temperature, $T_{\text {mould }}$ for mould temperature.

\subsection{Shrinkage Measurements}

In this work, we adopted width shrinkage as the quality parameter. This choice was made because width shrinkage is strongly dependent on the local solidification conditions and is less sensitive to the presence of constraints with respect to length shrinkage and to mould deformation with respect to thickness shrinkage. The shrinkage was defined as the relative difference between the mould and the product width (both evaluated at $25{ }^{\circ} \mathrm{C}$ ), as defined by the following equation:

$$
s_{i}=\left(d_{i}-d_{s i}\right) / d_{i},
$$

where $s$ was the shrinkage, $d s$ the sample local width, and $d$ the local cavity width (Fig. 1). The subscripts indicate the position inside the cavity where width shrinkage was measured, namely at the positions of pressure transducers inside the cavity (si indicates the transducer position $P_{i}$ ). This means that for each moulding condition, three results for shrinkage were obtained, each one related to a particular local history of temperature and pressure.

\section{FROM PRESSURE MEASUREMENTS TO SOLIDIFICATION PROFILE}

For amorphous polymers, it can be demonstrated [9] that, after the local solidification time $t_{\text {sol }}$, the local pressure profile follows an exponential law, namely:

$$
P=P_{\infty}+A \exp \left(-\frac{t}{\tau}\right)
$$

where $\tau$ is a the characteristic cooling time:

$$
\tau=\left(\frac{2}{\pi}\right)^{2} \frac{L^{2}}{\alpha} .
$$

In $\mathrm{Eq}(3), L$ is the local half-thickness and $\alpha$ is the thermal diffusivity of the material. The parameter $P_{\infty}$ in Eq. (2) is a constant, which represents the pressure that would be reached at very long times, when the polymer reaches thermal equilibrium with the mould. If $P_{\infty}$ is positive, it coincides with the residual pressure. However, it can also be negative, obviously losing any physical meaning, if the solid polymer detaches from the cavity walls.

If Eq. (2) holds true, a non-linear regression can be carried out on the experimental pressure curve, aimed at determining the value of $t_{\text {sol }}$ (i.e. the time after which the pressure curve is well described by an exponential curve as in Eq. (2)), and of $\tau$ (i.e. the characteristic time for that exponential curve).

The results of the procedure are shown in Figs. 2 and 3 for some of the moulding tests carried out. These figures show that after a few seconds, the experimental pressure evolutions are well described by the exponential curves at all positions and for all 
conditions. A sensitivity analysis showed that on changing the maximum allowed error between the best fitting curve and the experimental curve, the solidification times found by the procedure change by about $1 \mathrm{~s}$. The procedure captures the fact that by increasing the injection temperature, the solidification times generally increase. Furthermore, differences in the solidification times at the different positions were detected and it was found that, in most cases, the solidification took place at position $P_{4}$ (at the cavity tip) at earlier times than for positions $P_{2}$ and $P_{3}$, as expected on the basis of the fact that the temperature is higher closer to the injection point. As expected, it was also found that by increasing the cavity thickness the solidification times significantly increase.

The values found for $\tau$ are reported in Fig. 4 for all the pressure curves analysed in this work. All the values found for $\tau$ collapse around two numbers (indicated as horizontal dotted lines), one for the series obtained with the thicker cavity and one for the series obtained with the thinner cavity, which differ of a factor of about four. Considering the definition of $\tau$ (Eq. (3)), this fact is a confirmation of the reliability of the method since by doubling the thickness of the cavity the value of $\tau$ should indeed increase by a factor of four. Furthermore, the values found by the regression procedure are close to what can be calculated by substituting the value of thermal diffusivity ( $\alpha=10^{-7} \mathrm{~m}^{2} / \mathrm{s}[15]$ ) in Eq. (3), shown in Fig. 4 as horizontal solid lines.

An advantage of the procedure reported above is that it is possible to estimate the local solidification time without any knowledge of material properties, of moulding conditions, and even of local thickness. Thus, the procedure can be applied to a cavity of unknown or variable thickness.

Neglecting the variation in physical properties of the polymer with cooling and the effect of convection [9], the local solidification profile can be obtained:

$$
y_{s, \text { long }}^{*}(t) \simeq 1-\frac{2}{\pi} a \cos \left[\exp \left(\frac{t-t_{\text {sol }}}{\tau}\right)\right],
$$

in which $y^{*}$ is the normalized distance from the skin $\left(y^{*}=0\right.$ at the mould surface and $y^{*}=1$ at the midplane) and $y^{*}{ }_{S}$ identifies the layer which is solidifying at time $t\left(y^{*}{ }_{s}=1\right.$ for complete solidification and afterwards). The subscript long indicates that this solution is valid for Fourier numbers $\left(F_{O}=\alpha t / L^{2}\right)$ larger than 0.1, namely for longer times.

A solution for the heat conduction for shorter times can be obtained by the penetration theory. This allows the calculation of the solidification evolution inside the layers that solidify at short times $\left(F_{O}<0.1\right)$

$$
y_{s, s h o r t}^{*}(t) \simeq \operatorname{er} f^{-1}\left[\frac{4}{\pi} \exp \left(-\frac{t_{s o l}}{\tau}\right)\right] \sqrt{4\left(\frac{2}{\pi}\right)^{2} \frac{t}{\tau}} .
$$

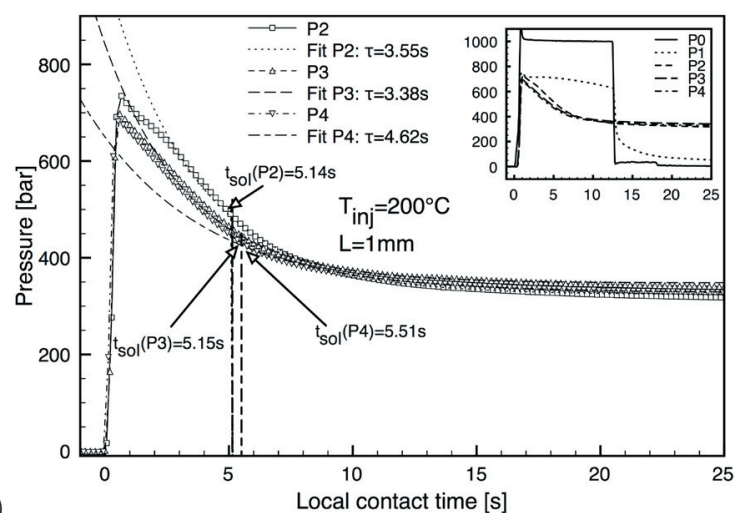

a)

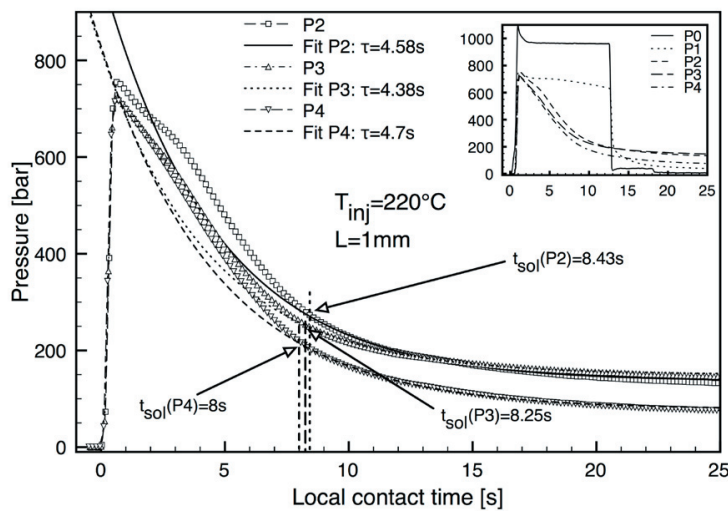

b)

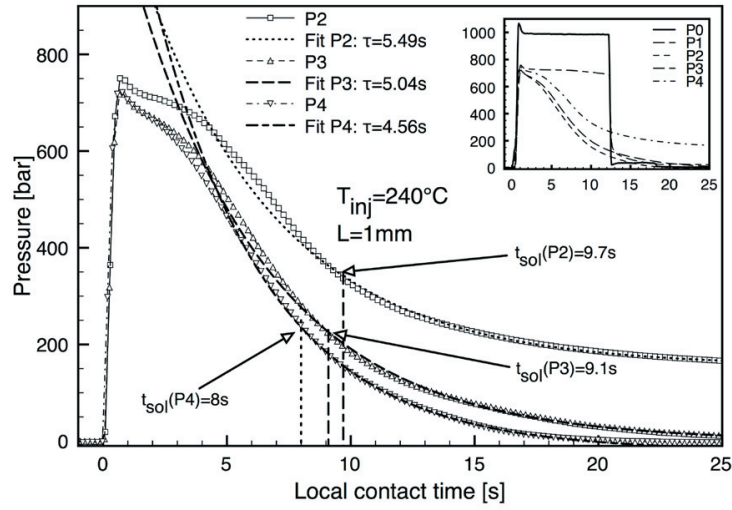

Fig. 2. Illustration of the exponential fitting on some of the pressure curves analysed in this work; $2 \mathrm{~mm}$ thick cavity; a) $T_{\text {inj }}=200^{\circ} \mathrm{C}$; b) $T_{\text {inj }}=220^{\circ} \mathrm{C}$; c) $T_{\text {inj }}=240{ }^{\circ} \mathrm{C}$

An equation that allows us to describe the solidification layer profile over the whole time range can be given as a combination of Eqs. (4) and (5) [9]: 


$$
y_{s}^{*}(t)=y_{s, \text { short }}^{*}(t)+\xi(t)\left[y_{s, \text { short }}^{*}(t)-y_{s, \text { long }}^{*}(t)\right],
$$

in which the function $\xi(t)$ should be zero at low Fourier numbers and 1 at high Fourier numbers.

Eq. (7) describes a transition from 0 to 1 in the neighbourhood of $F_{O}=0.1$.

One possible expression is:

$$
\xi(t)=1-\frac{1}{1+\exp \left[10\left(t-t_{c}\right)\right]},
$$

with

$$
t_{c}=0.1\left(\frac{\pi}{2}\right)^{2} \tau
$$

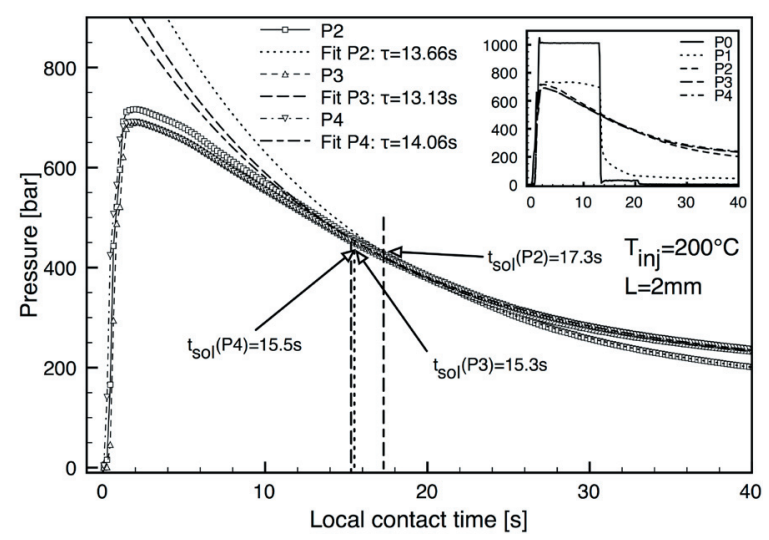

Fig. 3. Illustration of the exponential fitting on some of the pressure curves analysed in this work; $4 \mathrm{~mm}$ thick cavity

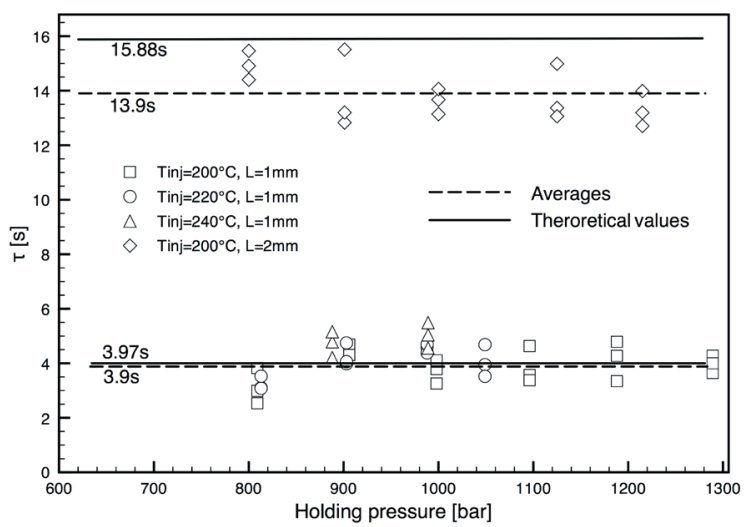

Fig. 4. Values of the parameter $T$ found for all the pressure curves analysed in this work; the horizontal lines identify the theoretical and the average values for $\tau$

Eqs. (4) to (8) show that knowledge of $t_{\text {sol }}$, namely the local solidification time, and of $\tau$, allows the estimation of the whole solidification profile. It is worth mentioning that the method does not require knowledge of the initial, mould or solidification temperatures, whose determination presents a certain degree of uncertainty, and it does not require any characterization of the physical parameters of the material.

\section{AVERAGE SOLIDIFICATION PRESSURE AND SHRINKAGE}

The definition of the average solidification pressure arises from considering a viscous - elastic model for shrinkage [16], namely from assuming that the polymer melt turns into an elastic solid as soon as it solidifies. Since solidification proceeds from the mould surfaces to the core, solidification pressure is different for each layer, thus each layer has a different stress-free configuration (larger dimensions for layers solidified under high pressure [16]). On ejection, each layer will experience a different stress so as to bring all of them to the same final length. On the basis of these considerations, the average value over the thickness of the pressures at which each layer solidifies, $\overline{P S}$, was introduced to take into account the effect of pressure on shrinkage.

$$
\overline{P S}=\int_{y^{*}=0}^{1} P(t) d y_{s}^{*}(t) .
$$

It was demonstrated [9], [11] and [16], that, for a given polymer, the average solidification pressure is directly related to the local shrinkage. However, the definition of the average solidification pressure requires knowledge of the temperature histories inside the polymer, in order to define $y_{s}^{*}(t)$. The main purpose of the present work is to define a suitable method for obtaining this piece of information using experimental local pressure alone.

Once the local solidification profile is known, the local average solidification pressure can be easily calculated by Eq. (9). As reported in the literature [9] and [16], local shrinkage should be directly correlated to $\overline{P S}$.

The procedure outlined above was then applied to all the pressure curves of each moulding test: first, the values of $t_{\text {sol }}$ and $\tau$ were obtained at each position and then the solidification profile was calculated. Eventually, the values of $\overline{P_{S}}$ were determined.

Fig. 5 shows the experimental values for the shrinkage measured at each cavity position and for all tests carried out on PS versus the local value of the average solidification pressure. Most of the shrinkage data are collected on a single plot, which confirms on the one hand the suitability of $\overline{P_{S}}$ in correlating to the quality of the moulded part and on the other 
hand the reliability of the procedure reported in this work in obtaining a single parameter, able to correlate with shrinkage, whose value can be determined by the experimental pressure evolution only. For the same average solidification pressure, the differences in shrinkage were less than $0.2 \%$ (it is worth recalling that the accuracy of measurement for shrinkage is $\pm 0.03 \%)$.

The procedure described in this work is suitable for a master-curve approach: a series of moulding tests can be carried out for the chosen material, recording the pressure curves and measuring the shrinkage close to the pressure transducer; for each pressure curve the value of $\overline{P_{S}}$ is calculated and a master curve of shrinkage vs. of $\overline{P S}$ is built; afterwards, the procedure is able to automatically associate a value for the shrinkage to each test by calculating on line the value of $\overline{P S}$ from each experimental pressure curve.

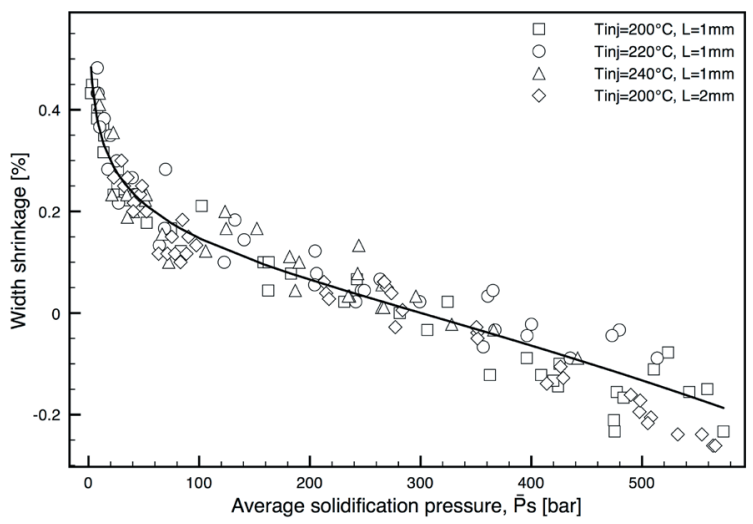

Fig. 5. Measured width shrinkage vs. average solidification pressure for each cavity position and for all tests carried out in this work

\section{CONCLUSIONS}

In this work a procedure was adopted to calculate the average solidification pressure, a parameter that is critical for the description of local shrinkage, by analysing the local pressure evolution measured using a conventional pressure transducer. The procedure was applied to a general purpose PolyStyrene, which was injection moulded under several processing conditions, where the cavity thickness was also changed. It was shown that all the shrinkage data collect on a single plot when reported versus the average solidification pressure calculated by analysing the experimental pressure curves. The procedure is thus suitable for a master-curve approach in which some data for the shrinkage versus the average solidification pressure can be used as a reference in order to estimate the shrinkage of the part by analysing the local pressure evolution. The described procedure is particularly suitable for on line monitoring of the chosen quality parameter and does not require either knowledge of local thickness or of the moulding conditions. Furthermore, it can be applied without any characterization of the physical parameters of the material.

\section{REFERENCES}

[1] Chen Z.B., Turng L.S. (2005). Current developments in process and quality control for injection molding. Advances in Polymer Technology, vol. 24, no. 3, p.165182, DOI:10.1002/adv.20046.

[2] Michaeli W., Schreiber A. (2009). Online control of the injection molding process based on process variables. Advances in Polymer Technology, vol. 28, no. 2, p. $65-$ 76, DOI:10.1002/adv.20153.

[3] Visvanathan, K., Balasubramaniam, K. (2007). Ultrasonic torsional guided wave sensor for flow front monitoring inside molds. Review of Scientific Instruments, vol. 78, no. 1, p. 015110, DOI:10.1063/1.2432258.

[4] Fung, K.T., Gao, F., Chen, X. (2007). Application of a capacitive transducer for online part weight prediction and fault detection in injection molding. Polymer Engineering \& Science, vol. 47, no. 4, p. 347-353, DOI:10.1002/pen.20700.

[5] Bur, T. (1998). Optical monitoring of polypropylene injection molding. Journal of Reinforced Plastics and Composites, vol. 17, no. 15, p. 1382-1390.

[6] De Santis, F., Pantani, R., Speranza V., Titomanlio, G. (2010). Analysis of shrinkage development of a semicrystalline polymer during injection molding. Industrial \& Engineering Chemistry Research, vol. 49, no. 5, p. 2469-2476, DOI:10.1021/ie901316p.

[7] Panchal, R.R., Kazmer, D.O. (2010). In-situ shrinkage sensor for injection molding. Journal of Manufacturing Science and Engineering, vol. 132, no. 6, p. 064503, DOI:10.1115/1.4002765.

[8] Kurt, M., Kamber, O.S., Kaynak, Y., Atakok, G., Girit, O. (2009). Experimental investigation of plastic injection molding: Assessment of the effects of cavity pressure and mold temperature on the quality of the final products. Materials \& Design, vol. 30, no. 8, p. 3217-3224, DOI:10.1016/j.matdes.2009.01.004.

[9] Speranza, V., Vietri, U., Pantani, R. (2011). Monitoring of injection molding of thermoplastics: Average solidification pressure as a key parameter for quality control. Macromolecular Research, vol. 19, no. 6, p. 542-554, DOI:10.1007/s13233-011-0610-9.

[10] Liu, S., Su, P., Lin, K. (2009). In-situ temperature measurements in the depths of injection molded parts. Measurement, vol. 42, no. 5, p. 771-777, DOI:10.1016/j.measurement.2009.01.002. 
[11] Speranza, V., Vietri, U., Pantani, R. (2012). Adopting the experimental pressure evolution to monitor online the shrinkage in injection molding. Industrial \& Engineering Chemistry Research, vol. 51, no. 49, p. 16034-16041, DOI:10.1021/ie302432v.

[12] Vietri, U., Sorrentino, A., Speranza, V., Pantani, R. (2011). Improving the predictions of injection molding simulation software. Polymer Engineering \& Science, vol. 51, no. 12, p. 2542 -2551, DOI:10.1002/pen. 22035.

[13] Sorrentino, A., Pantani, R. (2009). Pressure-dependent viscosity and free volume of atactic and syndiotactic polystyrene. Rheologica Acta, vol. 48, no. 4, p. 467478, DOI:10.1007/s00397-009-0348-x.

[14] Pantani, R., Speranza, V., Sorrentino, A., Titomanlio, G. (2002). Molecular orientation and strain in injection moulding of thermoplastics. Macromolecular Symposia, vol. 185, no. 1, p. 293-307, DOI:10.1002/1521-3900(200208)185:1<293::AIDMASY293>3.0.CO;2-8.

[15] Pantani, R., Speranza, V., Titomanlio, G. (2001). Relevance of mold-induced thermal boundary conditions and cavity deformation in the simulation of injection molding. Polymer Engineering \& Science, vol. 41, no. 11, p. 2022-2035, DOI:10.1002/ pen.10898.

[16] Jansen, K.M.B., Titomanlio, G. (1996). Effect of pressure history on shrinkage and residual stresses - Injection molding with constrained shrinkage. Polymer Engineering \& Science, vol. 36, no. 15, p. 2029-2040, DOI:10.1002/pen.10598. 